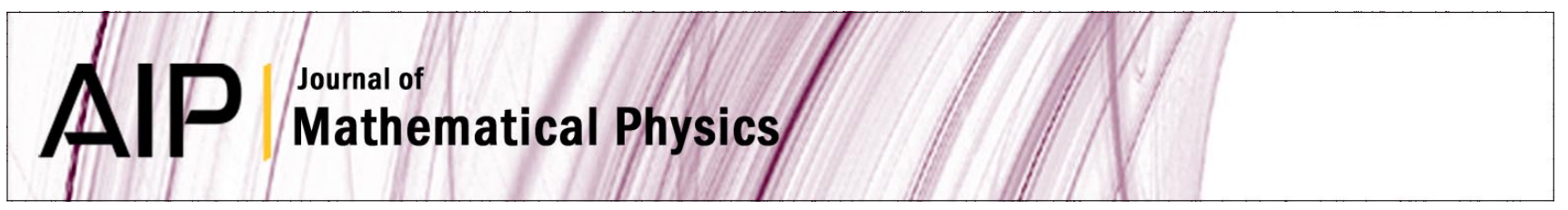

\title{
Approximately disentangling exponential operators
}

Daniel Scholz, Volodymyr G. Voronov, and Michael Weyrauch

Citation: J. Math. Phys. 51, 063513 (2010); doi: 10.1063/1.3413923

View online: http://dx.doi.org/10.1063/1.3413923

View Table of Contents: http://jmp.aip.org/resource/1/JMAPAQ/v51/i6

Published by the American Institute of Physics.

\section{Related Articles}

Dirac equation for generalized Pöschl-Teller scalar and vector potentials and a Coulomb tensor interaction by Nikiforov-Uvarov method

J. Math. Phys. 53, 022104 (2012)

Local trace formulae and scaling asymptotics for general quantized Hamiltonian flows

J. Math. Phys. 53, 023501 (2012)

A technique for physical realization of anisotropic density matrices with application to acoustic beam shifters

J. Appl. Phys. 111, 024907 (2012)

Circular law and arc law for truncation of random unitary matrix

J. Math. Phys. 53, 013301 (2012)

New symbolic tools for differential geometry, gravitation, and field theory

J. Math. Phys. 53, 013511 (2012)

\section{Additional information on J. Math. Phys.}

Journal Homepage: http://jmp.aip.org/

Journal Information: http://jmp.aip.org/about/about_the_journal

Top downloads: http://jmp.aip.org/features/most_downloaded

Information for Authors: http://jmp.aip.org/authors

\section{ADVERTISEMENT}

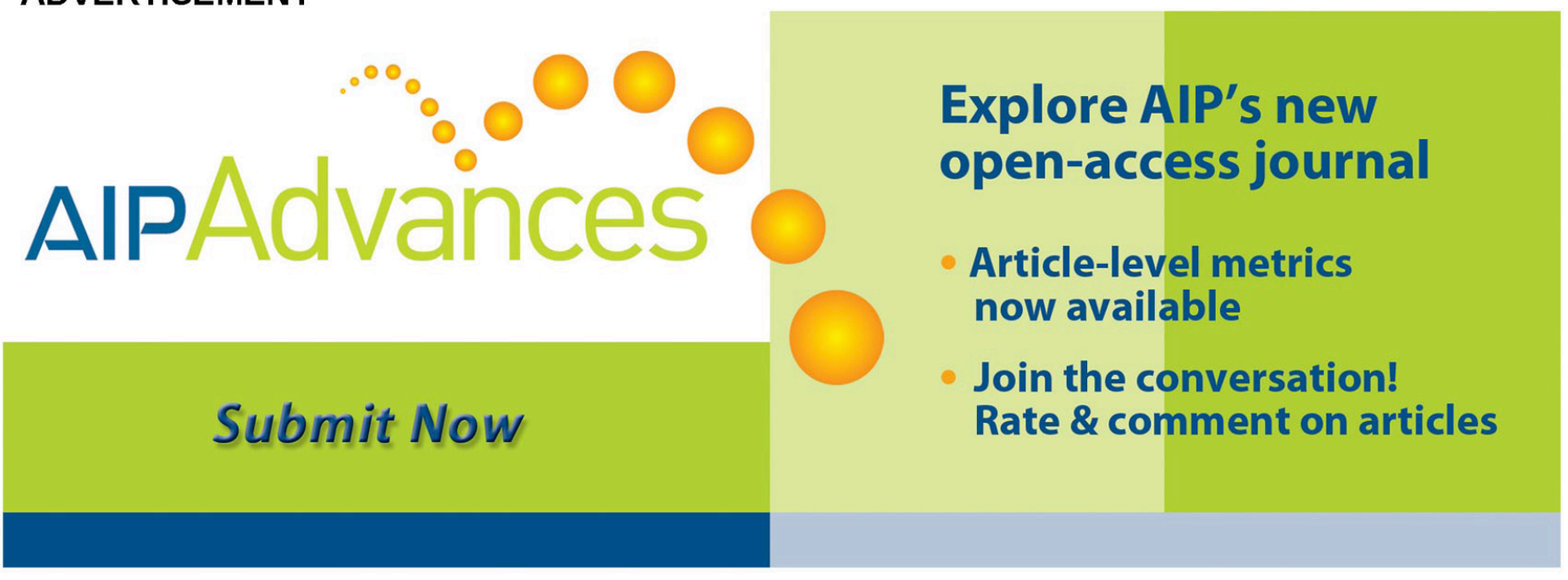




\title{
Approximately disentangling exponential operators
}

\author{
Daniel Scholz, ${ }^{1, a)}$ Volodymyr G. Voronov, ${ }^{2, b)}$ and Michael Weyrauch ${ }^{3, c)}$ \\ ${ }^{1}$ Institute for Numerical and Applied Mathematics, Georg-August-Universität Göttingen, \\ 37083 Germany \\ ${ }^{2}$ Faculty of Physics, Taras Shevchenko National University of Kyiv, 03022 Kyiv, Ukraine \\ ${ }^{3}$ Physikalisch-Technische Bundesanstalt, 38116 Braunschweig, Germany
}

(Received 21 October 2009; accepted 30 March 2010; published online 22 June 2010)

\begin{abstract}
A new method for the approximate disentangling of exponential operators based on the Baker-Campbell-Haussdorff theorem is suggested and implemented in a computer program. The operators to be disentangled must form a finite-dimensional Lie algebra. The accuracy of the method is tested and demonstrated in several explicitly calculated examples, where exact analytic solutions are available.
\end{abstract}

(C) 2010 American Institute of Physics. [doi:10.1063/1.3413923]

\section{INTRODUCTION}

The disentangling of exponential operators is a useful tool applied, e.g., in quantum mechanics, quantum field theory, optics, or physical chemistry. Mathematically, it may be regarded as a method for the solution of linear differential equations. ${ }^{1}$ The basic idea has been developed long ago, e.g., by Feynman ${ }^{2}$ and Glauber, ${ }^{3}$ and developed later into different directions by many authors (see, e.g., Refs. 1 and 4). The method was reviewed recently by Popov. ${ }^{5}$

The formulation of the disentangling problem is particularly straightforward if the operators to be disentangled are members of a finite-dimensional Lie algebra with generators $\left\{A_{1}, \ldots, A_{m}\right\}$. Then, under certain conditions discussed in more detail, e.g., in Ref. 1, it holds that

$$
\exp \left(\xi_{1} A_{1}+\cdots+\xi_{m} A_{m}\right)=\exp \left(\sigma_{1} A_{1}\right) \cdots \cdot \exp \left(\sigma_{m} A_{m}\right)
$$

with $\xi_{1}, \ldots, \xi_{m} \in \mathbb{C}$ given constants. Some of the $\xi_{i}$ may be zero. The disentangling problem to be solved is the determination of the $\sigma_{1}, \ldots, \sigma_{m} \in \mathbb{C}$ for a given Lie algebra $\left\{A_{1}, \ldots, A_{m}\right\}$. Here, we will assume that for the Lie algebra under consideration the relation (1) holds at least locally, and mathematical questions on the (global) existence of Eq. (1) will not be addressed. ${ }^{6}$ The aim of the present paper is to suggest a new practical way for the approximate determination of the complex constants $\sigma_{1}, \ldots, \sigma_{m} \in \mathrm{C}$ and to provide a suitable computer implementation.

There are various techniques that solve the disentanglement problem [Eq. (1)] for certain cases analytically and exactly. We briefly mention three of them: parameter differentiation, matrix representation of the Lie algebra, and a method using similarity transformations.

Parameter differentiation was first used by Glauber ${ }^{3}$ and exposed in detail by Wilcox. ${ }^{4}$ First one introduces a parameter $t$ into Eq. (1),

$$
\exp \left(t\left(\xi_{1} A_{1}+\cdots+\xi_{m} A_{m}\right)\right)=\exp \left(\sigma_{1}(t) A_{1}\right) \cdots \cdot \exp \left(\sigma_{m}(t) A_{m}\right)
$$

and then differentiates this equation with respect to $t$. Using the well known relation

\footnotetext{
${ }^{\text {a)} E l e c t r o n i c ~ m a i l: ~ d s c h o l z @ m a t h . u n i-g o e t t i n g e n . d e . ~}$

b)Electronic mail: v_voronov@univ.kiev.ua.

${ }^{c}$ Electronic mail: michael.weyrauch@ptb.de.
} 


$$
\exp (A) \cdot B \exp (-A)=\sum_{k=0}^{\infty} \frac{1}{k !} \cdot\{A, B\}_{k}
$$

with the nested commutators $\{A, B\}_{k}=\left[A,\{A, B\}_{k-1}\right]$ and $\{A, B\}_{0}=B$ as well as the assumed Lie algebra structure in order to calculate the nested commutators finally leads to a system of ordinary differential equations. If this system can be solved analytically, one may be able to obtain the solutions for $\sigma_{1}$ to $\sigma_{m}$ in closed form. Examples are discussed by Wilcox. ${ }^{4}$

A purely algebraic method based on a matrix representation of the Lie algebra may be useful if such a representation can be easily obtained. If the exponential of these matrices can be calculated in closed form, one obtains a system of nonlinear equations whose solutions yield the coefficients $\sigma_{1}$ to $\sigma_{m}$. Examples from quantum optics are presented in Refs. 7 and 8.

A method already suggested by Wilcox ${ }^{4}$ and exposed in more detail by DasGupta ${ }^{9}$ compares similarity transformations induced by the left hand (entangled) side of Eq. (1) and the right hand (disentangled) side of Eq. (1) on the generators of the Lie algebra. Calculations make extensive use of Eq. (3) and lead to a system of nonlinear equations in the unknown $\sigma_{1}$ to $\sigma_{m}$. Note that if the identity operator $I$ is a generator of the given Lie algebra, the similarity transformation method cannot compute the corresponding coefficient.

The approximation method proposed here uses the Baker-Campbell-Haussdorff (BCH) theorem $^{10-12}$ in order to rewrite the right hand side of Eq. (1). The $\mathrm{BCH}$ theorem asserts that the product of the exponentials of two noncommutative variables $A$ and $B$ may be expressed as the exponential of an infinite sum

$$
\exp (A) \cdot \exp (B)=\exp \left(A+B+\sum_{n=2}^{\infty} Z_{n}\right)
$$

where the $\mathrm{BCH}$ term $Z_{n}$ may be expressed as a linear combination of nested commutators of the noncommuting variables $A$ and $B$. If we truncate the sum in Eq. (4) at $n=p$, we obtain the $\mathrm{BCH}$ approximation of order $p$ for the product of two exponentials.

The $\mathrm{BCH}$ approximation for the disentangling of exponential operators is developed in detail in Sec. II. A computer implementation is provided in Sec. III, followed by a number of examples (Sec. IV) in order to demonstrate the accuracy of the method by comparison with known exact results. Conclusions in Sec. V summarize the paper.

\section{BCH APPROXIMATION}

We assume that we are given a Lie algebra with generators $A_{1}, \ldots, A_{m}$ satisfying the commutation relations

$$
\left[A_{i}, A_{j}\right]=a_{1}^{i j} \cdot A_{1}+\cdots+a_{m}^{i j} \cdot A_{m}=\left\{a_{1}^{i j}, \ldots, a_{m}^{i j}\right\}
$$

with the structure constants $a_{k}^{i j} \in \mathrm{C}$ for $k=1, \ldots, m$ and $1 \leq i<j \leq m$. The component notation for a Lie element is implicitly introduced after the second equal sign in Eq. (5). It is now our goal to find an approximation for the coefficients $\sigma_{1}, \ldots, \sigma_{m}$ in order to disentangle the exponential of a Lie element $\xi=\left\{\xi_{1}, \ldots, \xi_{m}\right\}$,

$$
\exp \left(\xi_{1} A_{1}+\cdots+\xi_{m} A_{m}\right)=\exp \left(\sigma_{1} A_{1}\right) \cdot \cdots \cdot \exp \left(\sigma_{m} A_{m}\right)
$$

The approximation is obtained as follows. In order to combine the exponentials on the right hand side of Eq. (6), we use a BCH approximation of order $p$ repeatedly [see explanation after Eq. (4)]. Then, using the structure of the Lie algebra [Eq. (5)], it is possible to evaluate the nested commutators in the resulting exponential and, in principle, write the right hand side of Eq. (6) in the form 


$$
\exp \left(\sigma_{1} A_{1}\right) \cdots \cdot \exp \left(\sigma_{m} A_{m}\right) \approx \exp \left(f_{1}^{p}\left(\sigma_{1}, \ldots, \sigma_{m}\right) \cdot A_{1}+\cdots+f_{m}^{p}\left(\sigma_{1}, \ldots, \sigma_{m}\right) \cdot A_{m}\right),
$$

where $f_{1}^{p}, \ldots, f_{m}^{p}: \mathrm{C}^{m} \rightarrow \mathrm{C}$ are functions that depend on the order of $p$ of the $\mathrm{BCH}$ approximation.

In order to obtain an approximation for $\sigma_{i}$, one finally needs to solve the system of nonlinear equations

$$
F^{p}\left(\tau_{1}, \ldots, \tau_{m}\right)=\left(\begin{array}{c}
f_{1}^{p}\left(\tau_{1}, \ldots, \tau_{m}\right) \\
\vdots \\
f_{m}^{p}\left(\tau_{1}, \ldots, \tau_{m}\right)
\end{array}\right)=\left(\begin{array}{c}
\xi_{1} \\
\vdots \\
\xi_{m}
\end{array}\right) .
$$

Let $\left(\tau_{1}, \ldots, \tau_{m}\right)$ be a solution of this system. We then obtain the approximation $\sigma_{k} \approx \tau_{k}$ for $k=1, \ldots, m$. Note that the functions $f_{k}^{p}\left(\tau_{1}, \ldots, \tau_{m}\right)$ for $k=1, \ldots, m$ are polynomials of degree $p-1$.

While the procedure outlined above is conceptually straightforward, a practical implementation requires a few tools. First we need a way to determine the $\mathrm{BCH}$ terms up to the desired order. A polynomial representation of the $\mathrm{BCH}$ term $Z_{n}$ defined in Eq. (4) is given by a weighted sum of the $2^{n}$ "words" of length $n$, which can be written with two letters $A$ and $B$,

$$
Z_{n}=\sum_{s=1}^{2^{n}} \Lambda_{n}^{s} \cdot X_{1}^{s} \cdots X_{n}^{s}
$$

Here, in each word $X_{1}^{s} \cdots X_{n}^{s}, X_{i}^{s}$ stands for a factor $A$ or $B$. Practical methods for the calculation of the coefficients $\Lambda_{n}^{s}$ are reviewed and developed in Ref. 13. It holds that $\Lambda_{1}^{1}=\Lambda_{1}^{2}=1$ and $\Lambda_{n}^{s} \in \mathbb{Q}$ for all $s=1, \ldots, 2^{n}$. For example, for $Z_{3}$ one obtains

$$
Z_{3}=\frac{1}{12} A A B-\frac{1}{6} A B A+\frac{1}{12} A B B+\frac{1}{12} B A A-\frac{1}{6} B A B+\frac{1}{12} B B A .
$$

In order to make use of the Lie algebra structure assumed above, the polynomial representation of the $\mathrm{BCH}$ terms must be converted into a representation in terms of nested commutators. Such a representation is known to exist, but it is not unique. A suitable map $\Psi$ from polynomials to nested commutators was first developed by Dynkin, ${ }^{14,15}$

$$
\Psi\left(Z_{n}\right)=\frac{1}{n} \cdot \sum_{s=1}^{2^{n}} \Lambda_{n}^{s} \cdot\left[\left[\cdots\left[\left[\left[X_{1}^{s}, X_{2}^{s}\right], X_{3}^{s}\right], X_{4}^{s}\right], \ldots\right], X_{n}^{s}\right] .
$$

Later a map $\Phi$ that yields fewer terms than the Dynkin map was conjectured by Oteo, ${ }^{16}$ and we recently proved that this map is valid. ${ }^{13}$ The map $\Phi$ is defined by

$$
\Phi\left(Z_{n}\right)=\sum_{\substack{s=1 \\ X_{1}^{s}=A, X_{2}^{s}=B}}^{2^{n}} \frac{\Lambda_{n}^{s}}{N\left(X_{1}^{s}, \ldots, X_{n}^{s}\right)} \cdot\left[\left[\cdots\left[\left[[A, B], X_{3}^{s}\right], X_{4}^{s}\right], \ldots\right], X_{n}^{s}\right] .
$$

Here, $N\left(X_{1}^{s}, \ldots, X_{n}^{s}\right)$ is the number of $A$ 's in the word $X_{1}^{s} \cdots X_{n}^{s}$, e.g., $N(A B A A B)=3$. We proved in Ref. 13 the following theorem:

For all $n \geq 2$, it holds that $\Phi\left(Z_{n}\right)=Z_{n}$.

For example, for $Z_{3}$ one obtains

$$
\begin{aligned}
Z_{3} & =\frac{1}{12} A A B-\frac{1}{6} A B A+\frac{1}{12} A B B+\frac{1}{12} B A A-\frac{1}{6} B A B+\frac{1}{12} B B A \\
& =-\frac{1}{12}[[A, B], A]+\frac{1}{12}[[A, B], B]=\Phi\left(Z_{3}\right) .
\end{aligned}
$$

Alternatively, with the Dynkin map $\Psi$, one would obtain 


$$
Z_{3}=-\frac{1}{18}[[A, B], A]+\frac{1}{36}[[A, B], B]+\frac{1}{36}[[B, A], A]-\frac{1}{18}[[B, A], B]=\Psi\left(Z_{3}\right),
$$

which is, however, less convenient for practical calculations.

With the tools collected above, it is possible to explicitly calculate the function $F^{p}$ in Eq. (8) to the desired order $p$ with the help of a computer. Finally, we need a suitable method to solve the system of nonlinear equations given in Eq. (8). In general, there are several solutions of such a polynomial system, and we need to pick out the correct one. Our procedure to do this is based on the following observation: each solution $\tau=\left\{\tau_{1}, \tau_{2}, \ldots, \tau_{m}\right\}$ of the set of equations, of course, depends on the parameters $\xi=\left\{\xi_{1}, \xi_{2}, \ldots, \xi_{m}\right\}$. However, for $\xi=e_{1}=\{1,0, \ldots, 0\}$ the solution must be $\tau_{1}=\xi_{1}, \tau_{2}=0, \ldots, \tau_{m}=0$, according to Eq. (6). Therefore, as a function of $\xi$ a solution $\tau(\xi)$ must fulfill the boundary condition $\tau\left(e_{1}\right)=\left\{\xi_{1}, 0, \ldots, 0\right\}$. Our procedure constructs the function $\tau(\xi)$ starting from this boundary, i.e., we start from the trivial solution for $\xi=e_{1}$ and then solve several auxiliary problems

$$
F^{p}(\tau)=t \cdot \xi+(1-t) \cdot e_{1} \text { for } t=\frac{1}{M}, \frac{2}{M}, \ldots, \frac{M-1}{M}, 1,
$$

where the parameter $t$ controls the "distance" of the auxiliary problem to the problem we want to solve $(t=1)$. The parameter $M$ defines the number of auxiliary problems to be considered.

For each step in this procedure, we solve the nonlinear system of Eq. (8) using Newton's iteration method

$$
\tau^{k+1}=\tau^{k}-\left(D F\left(\tau^{k}\right)\right)^{-1} \cdot\left(F\left(\tau^{k}\right)-\xi\right)
$$

with $\operatorname{DF}^{p}\left(\tau_{1}, \ldots, \tau_{m}\right)$ denoting the Jacobian of $F^{p}\left(\tau_{1}, \ldots, \tau_{m}\right)$. As the starting vector $\tau^{0}$ for each Newton iteration, we use the solution of the previous step starting with $\tau^{0}=e_{1}$ for $t=1 / M$. In summary, we suggest the following solution approach.

Input: Absolute accuracy $\varepsilon>0$ and number of auxiliary problems $M \in \mathbb{N}$

1. Set $\tau:=e_{1}, \hat{\tau}:=e_{1}, k:=0$, and $t:=0$.

2. If $k=M$, stop. Else set $k=k+1$ and $t=k / M$.

3. Set $\tau=\hat{\tau}$ and $\hat{\tau}=\tau-(D F(\tau))^{-1} \cdot\left(F(\tau)-\left(t \cdot \xi+(1-t) \cdot e_{1}\right)\right)$.

4. If $\|\tau-\hat{\tau}\|_{2}<\varepsilon$, go to Step 2. Else go to Step 3 .

Output: Approximation $\hat{\tau}$.

In all examples in Sec. IV we applied this technique using $\varepsilon=10^{-10}$ and $M=10$.

\section{COMPUTER IMPLEMENTATION}

In this section we provide a MATHEMATICA7 (Ref. 17) implementation of the BCH approximation method outlined in Sec. II. Of course, similar implementations are easily possible in other languages.

The implementation assumes that the commutation relations of the Lie algebra are coded as $\mathrm{K}[i, j]=\left\{\mathrm{a}_{-} \mathcal{1}^{\wedge}\{i j\}, \ldots, \mathrm{a}_{-} \mathrm{m}\{i j\}\right\}$ for all $1 \leq i<j \leq m$. For example, for the $\mathrm{SU}(1,1)$ algebra with generators $\left\{K_{+}, K_{0}, K_{-}\right\}$, which is defined by the commutation relations

$$
\left[K_{+}, K_{0}\right]=-K_{+}, \quad\left[K_{0}, K_{-}\right]=-2 K_{0}, \quad\left[K_{0}, K_{-}\right]=-K_{-},
$$

one needs to enter the following MATHEMATICA statements:

$$
\mathrm{K}[1,2]=\{-1,0,0\} ; \mathrm{K}[1,3]=\{0,-2,0\} ; \mathrm{K}[2,3]=\{0,0,-1\} ;
$$


The commutator of two Lie elements $a$ and $\mathrm{b}$ is then calculated by the following function:

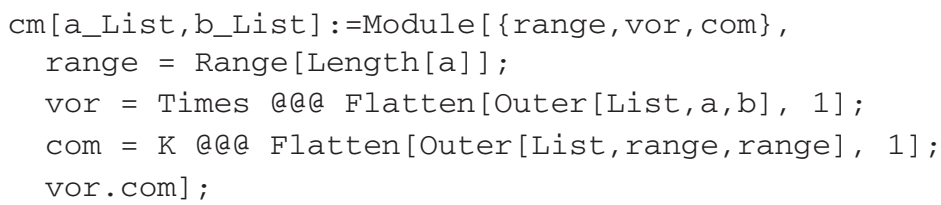

Using again the $\mathrm{SU}(1,1)$ algebra as an example, the Lie element $2 K_{0}+K_{-}$is coded by $\{0,2,1\}$ and the Lie element $K_{+}+K_{-}$is coded by $\{1,0,1\}$. Hence, for example, the input $\mathrm{Cm}[\{0,2,1\},\{1,0,1\}]$ produces $2 * \mathrm{~K}[2,1]+2 * \mathrm{~K}[2,3]+\mathrm{K}[3,1]+\mathrm{K}[3,3]$, which simplifies to $\{2,2,-2\}$.

Nested commutators are computed by the code

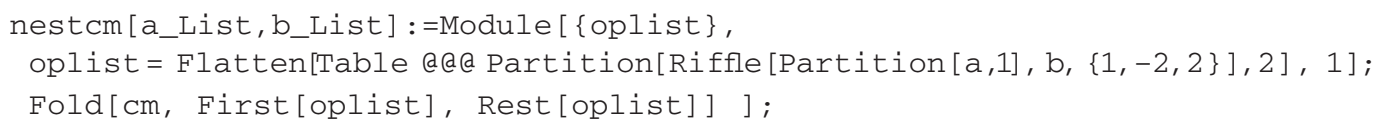

We compute the $\mathrm{BCH}$ terms up to approximation order $p$ using Goldberg's method. ${ }^{18} \mathrm{~A}$ slightly different variant of the code below is discussed and documented in more detail in Ref. 13.

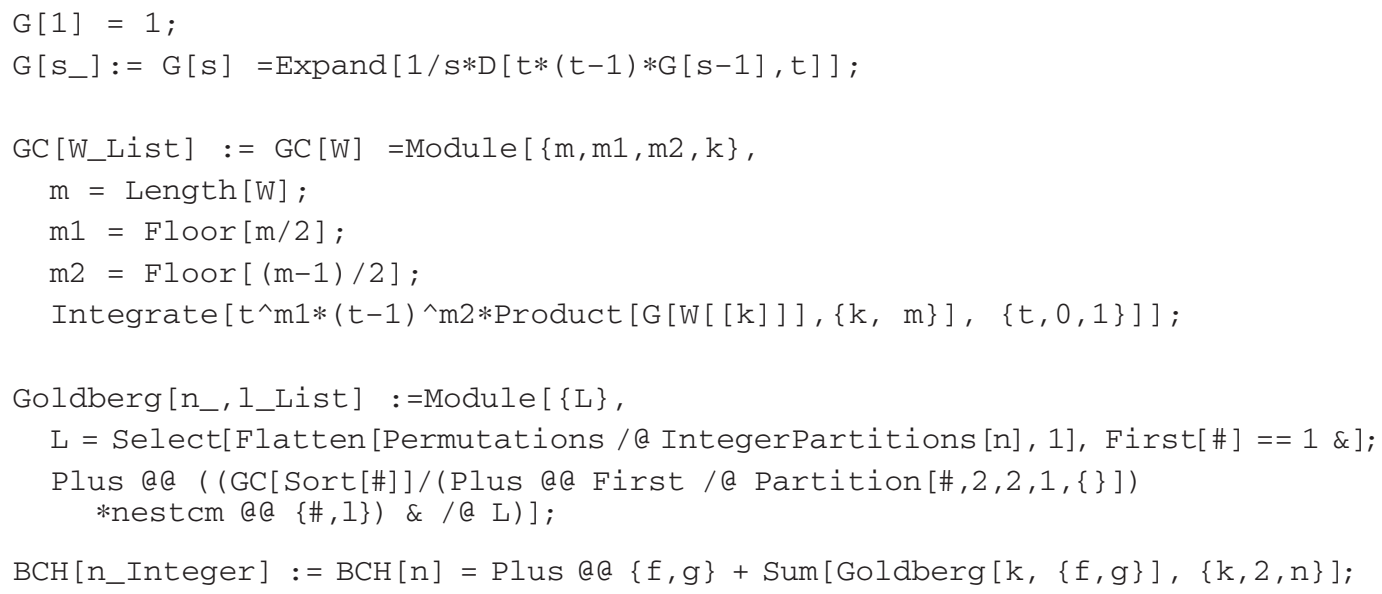

$\mathrm{BCH}[\mathrm{p}]$ returns all $\mathrm{BCH}$ terms up to the order of $p$ in a commutator representation for noncommutating variables $\mathrm{f}$ and $\mathrm{g}$. Here, unlike in Ref. 13, we directly applied the map $\Phi$ as defined in Sec. II.

The set of nonlinear equations (8) is set up and solved using the command sigmas. The noncommuting variables $\mathrm{f}$ and $\mathrm{g}$ in the $\mathrm{BCH}$ terms are replaced by the appropriate Lie elements:

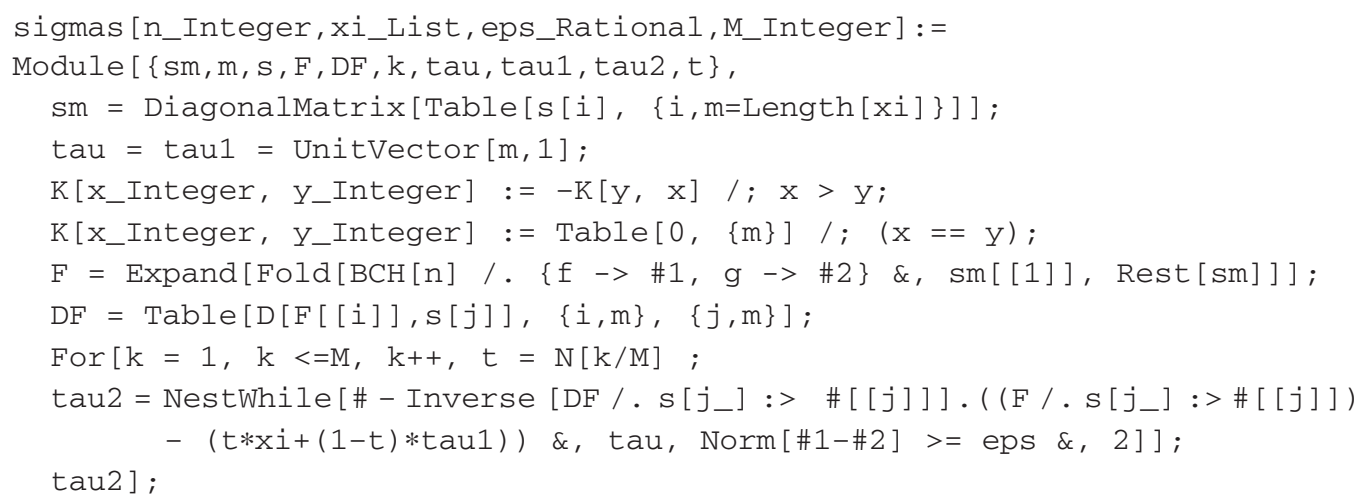


TABLE I. Comparison of exact and approximate disentangling coefficients for the Lie algebra $\{A, B\}$ with $[A, B]=\lambda B$ defined in Sec. IV A.

\begin{tabular}{cccc}
\hline \hline$p$ & $\{\alpha, \beta, \lambda\}$ & $\left\{\left\|\tau_{1}-\sigma_{1}\right\|,\left\|\tau_{2}-\sigma_{2}\right\|\right\}$ & CPU time (s) \\
\hline 4 & $\{1,1,1\}$ & $\left\{0,5.41 \times 10^{-4}\right\}$ & $<0.1$ \\
8 & $\{1,1,1\}$ & $\left\{0,3.22 \times 10^{-7}\right\}$ & $<0.1$ \\
12 & $\{1,1,1\}$ & $\left\{0,2.06 \times 10^{-10}\right\}$ & 1.0 \\
4 & $\{1+i, 1-i, 1\}$ & $\left\{0,2.90 \times 10^{-3}\right\}$ & $<0.1$ \\
8 & $\{1+i, 1-i, 1\}$ & $\left\{0,6.89 \times 10^{-6}\right\}$ & $<0.1$ \\
12 & $\{1+i, 1-i, 1\}$ & $\left\{0,1.76 \times 10^{-8}\right\}$ & 1.0 \\
4 & $\left\{\frac{1}{2}, 1-i, 2\right\}$ & $\left\{0,7.65 \times 10^{-4}\right\}$ & $<0.1$ \\
8 & $\left\{\frac{1}{2}, 1-i, 2\right\}$ & $\left\{0,4.56 \times 10^{-7}\right\}$ & $<0.1$ \\
12 & $\left\{\frac{1}{2}, 1-i, 2\right\}$ & $\left\{0,2.91 \times 10^{-10}\right\}$ & 1.0 \\
\hline \hline
\end{tabular}

The variable $\mathrm{F}$ holds the function $F^{p}\left(\sigma_{1}, \ldots, \sigma_{m}\right)$ and the rest of the code implements Newton's iteration method, as described in Sec. II.

Thus, the function sigmas $[\mathrm{p}, \mathrm{xi}, \mathrm{epsilon}, \mathrm{M}]$ returns the desired approximation for the coefficients $\sigma_{1}$ to $\sigma_{m}$. Here, $\mathrm{p}$ is the $\mathrm{BCH}$ approximation order, $\mathrm{xi}$ is the vector $\xi=\left(\xi_{1}, \ldots, \xi_{m}\right)$ as given in Eq. (1), and epsilon and M are the parameters $\varepsilon$ and $M$, which control Newton's iteration method, as described in Sec. II.

\section{EXAMPLES}

In order to study the applicability of our implementation of the $\mathrm{BCH}$ approximation for the disentangling of exponential operators, we here present a few numerical examples. For all examples, analytical results are available for comparison. Our program was run on a standard personal computer with $1.8 \mathrm{GHz}$ and 1024 Mbytes of memory. The CPU times in Tables I-V correspond to this machine.

\section{A. Two-dimensional Lie algebra}

Consider the Lie algebra $\{A, B\}$ with $[A, B]=\lambda B$ and $\lambda \in \mathrm{C}$. We look for $\sigma_{1}, \sigma_{2} \in \mathrm{C}$ such that

$$
\exp (\alpha A+\beta B) \approx \exp \left(\sigma_{1} A\right) \cdot \exp \left(\sigma_{2} B\right) .
$$

As shown, e.g., in Ref. 9 by the similarity transformation method mentioned in Sec. I, it holds that

$$
\sigma_{1}=\alpha \quad \text { and } \quad \sigma_{2}=\frac{\beta}{\lambda \alpha} \cdot\left(1-\mathrm{e}^{-\lambda \alpha}\right) .
$$

Using the $\mathrm{BCH}$ approximation up to order $p=4$, one finds

$$
\begin{gathered}
\exp \left(\sigma_{1} A\right) \cdot \exp \left(\sigma_{2} B\right) \approx \exp \left(\sigma_{1} A+\sigma_{2} B+\frac{1}{2} \sigma_{1} \sigma_{2} \lambda B+\frac{1}{12} \sigma_{1}^{2} \sigma_{2} \lambda^{2} B\right) \\
=\exp \left(\sigma_{1} \cdot A+\sigma_{2}\left(1+\frac{1}{2} \sigma_{1} \lambda+\frac{1}{12} \sigma_{1}^{2} \lambda^{2}\right) \cdot B\right) .
\end{gathered}
$$

Hence, we obtain

$$
F^{4}\left(\tau_{1}, \tau_{2}\right)=\left(\begin{array}{c}
\tau_{1} \\
\tau_{2}\left(1+\frac{1}{2} \tau_{1} \lambda+\frac{1}{12} \tau_{1}^{2} \lambda^{2}\right)
\end{array}\right)=\left(\begin{array}{l}
\alpha \\
\beta
\end{array}\right),
$$

and the solution of this system is 


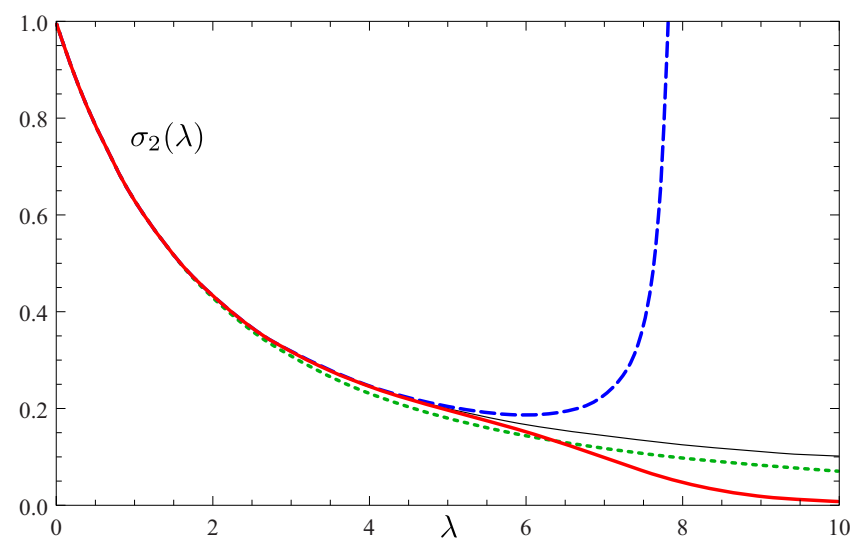

FIG. 1. (Color online) Comparison of exact and approximate disentangling coefficient $\sigma_{2}$ for the two-dimensional Lie algebra $\{A, B\}$ defined in Sec. IV A for $0 \leq \lambda \leq 10$. Exact solution (thin black curve), approximation order $p=4$ (dotted green curve), approximation order $p=9$ (dashed blue curve), and approximation order $p=12$ (solid red curve).

$$
\tau_{1}=\alpha \quad \text { and } \quad \tau_{2}=\frac{\beta}{1+\frac{1}{2} \alpha \lambda+\frac{1}{12} \alpha^{2} \lambda^{2}} .
$$

Table I presents numerical results for different approximation orders $p$ and selected values for $\alpha$, $\beta$, and $\lambda$.

For the present example, it is furthermore possible to address questions of convergence. Comparing the exact result for $\sigma_{2}$ [Eq. (20)] with the $\mathrm{BCH}$ approximation $\tau_{2}$ [Eq. (24)], one asserts that the denominator in Eq. (24) results from the expansion

$$
\frac{x}{1-\exp (-x)}=\sum_{n=0}^{\infty} B_{n} \frac{(-x)^{n}}{n !}=1+\frac{1}{2} x+\frac{1}{12} x^{2}-\frac{1}{720} x^{4}+\cdots,
$$

which is a relation well known from one possible definition of the Bernoulli numbers $B_{n}$. It is known that this series converges for $0<|x|<2 \pi$, which in this case defines the radius of convergence for our method to work. Specifically, it must hold that $0<|\alpha \lambda|<2 \pi$. In Fig. 1, we show numerical results for $\alpha=\beta=1$ and $0 \leq \lambda \leq 10$. The exact solution for $\sigma_{2}=\sigma_{2}(\lambda)$ corresponds to the thin black curve, and the $\mathrm{BCH}$ approximation for orders $p=4, p=9$, and $p=12$ are illustrated by the dotted, dashed, and solid curves, respectively. It can be seen that one cannot expect convergence for values $\lambda \geq 2 \pi$.

\section{B. Four-dimensional Lie algebra}

In our second example we consider the Lie algebra $\{A, B, D, I\}$ with the commutation relations

$$
[A, B]=-\lambda D, \quad[A, D]=-\mu B, \quad[A, I]=0, \quad[B, D]=-\alpha I, \quad[B, I]=0, \quad[D, I]=0
$$

for $\lambda, \mu, \alpha \in \mathrm{C}$. Our goal is to approximate the coefficients $\sigma_{1}, \ldots, \sigma_{4} \in \mathrm{C}$ such that

$$
\exp (A+B) \approx \exp \left(\sigma_{1} A\right) \cdot \exp \left(\sigma_{2} B\right) \cdot \exp \left(\sigma_{3} D\right) \cdot \exp \left(\sigma_{4} I\right) .
$$

We remark that, in general, every ordering of the exponentials would yield different disentanglement coefficients $\sigma_{i}$.

Exact solutions are, e.g., given in Ref. 19,

$$
\sigma_{1}=1, \quad \sigma_{2}=\frac{\sinh (\sqrt{\lambda \mu})}{\sqrt{\lambda \mu}}, \quad \sigma_{3}=\frac{\cosh (\sqrt{\lambda \mu})-1}{\mu}, \quad \sigma_{4}=\frac{\alpha}{4 \mu} \cdot\left(\frac{\sinh (2 \sqrt{\lambda \mu})}{\sqrt{\lambda \mu}}-2\right) .
$$

The $\mathrm{BCH}$ approximation up to order $p=4$ yields the system of equations 
TABLE II. Comparison of exact and approximate disentangling coefficients for the four-dimensional Lie algebra defined in Sec. IV B.

\begin{tabular}{cccc}
\hline \hline$p$ & $\{\lambda, \mu, \alpha\}$ & $\left\{\left\|\tau_{1}-\sigma_{1}\right\|, \ldots,\left\|\tau_{4}-\sigma_{4}\right\|\right\}$ & CPU time (s) \\
\hline 4 & $\{1,1,1\}$ & $\left\{0,2.27 \times 10^{-3}, 1.73 \times 10^{-3}, 9.96 \times 10^{-4}\right\}$ & $<0.1$ \\
8 & $\{1,1,1\}$ & $\left\{0,1.35 \times 10^{-6}, 1.03 \times 10^{-6}, 2.34 \times 10^{-6}\right\}$ & 0.3 \\
12 & $\{1,1,1\}$ & $\left\{0,8.64 \times 10^{-10}, 6.58 \times 10^{-10}, 2.62 \times 10^{-9}\right\}$ & 7.2 \\
4 & $\left\{1-\frac{i}{2}, \frac{1}{2}+i, 2\right\}$ & $\left\{0,3.64 \times 10^{-3}, 3.03 \times 10^{-3}, 6.60 \times 10^{-3}\right\}$ & $<0.1$ \\
8 & $\left\{1-\frac{i}{2}, \frac{1}{2}+i, 2\right\}$ & $\left\{0,3.38 \times 10^{-6}, 2.82 \times 10^{-6}, 1.23 \times 0^{-5}\right\}$ & 0.3 \\
12 & $\left\{1-\frac{i}{2}, \frac{1}{2}+i, 2\right\}$ & $\left\{0,3.37 \times 10^{-9}, 2.81 \times 10^{-9}, 1.95 \times 10^{-8}\right\}$ & 7.8 \\
4 & $\left\{2, \frac{i}{2},-1\right\}$ & $\left\{0,1.50 \times 10^{-3}, 2.80 \times 10^{-3}, 6.96 \times 10^{-3}\right\}$ & $<0.1$ \\
8 & $\left\{2, \frac{i}{2},-1\right\}$ & $\left\{0,8.93 \times 10^{-7}, 1.66 \times 10^{-6}, 7.36 \times 10^{-6}\right\}$ & 0.3 \\
12 & $\left\{2, \frac{i}{2},-1\right\}$ & $\left\{0,5.71 \times 10^{-10}, 1.06 \times 10^{-9}, 6.76 \times 10^{-9}\right\}$ & 7.6 \\
\hline \hline
\end{tabular}

$$
F^{4}\left(\tau_{1}, \tau_{2}, \tau_{3}, \tau_{4}\right)=\left(\begin{array}{c}
\tau_{1} \\
\tau_{2}-\frac{1}{2} \tau_{1} \tau_{3} \mu+\frac{1}{12} \tau_{1}^{2} \tau_{2} \lambda \mu \\
\tau_{3}-\frac{1}{2} \tau_{1} \tau_{2} \lambda+\frac{1}{12} \tau_{1} \tau_{3} \mu \lambda \\
\tau_{4}-\frac{1}{2} \tau_{2} \tau_{3} \alpha-\frac{1}{12} \tau_{1} \tau_{2}^{2} \lambda \alpha+\frac{1}{12} \tau_{1} \tau_{3}^{2} \mu \alpha
\end{array}\right)=\left(\begin{array}{l}
1 \\
1 \\
0 \\
0
\end{array}\right) .
$$

Numerical comparison between the exact solution and the $\mathrm{BCH}$ approximation can be found in Table II.

\section{C. $S U(1,1)$ algebra}

Here and in the following examples we will use the same notations as given in Refs. 7 and 9. The SU(1,1) Lie algebra $\left\{K_{+}, K_{0}, K_{-}\right\}$satisfies the commutation relations

$$
\left[K_{+}, K_{0}\right]=-K_{+}, \quad\left[K_{+}, K_{-}\right]=-2 K_{0}, \quad\left[K_{0}, K_{-}\right]=-K_{-} .
$$

For complex parameters $\alpha$, denote by $\alpha^{*}$ the conjugate of $\alpha$. Our aim is to approximate the disentanglement coefficients $\sigma_{1}$ to $\sigma_{3}$ in

$$
\exp \left(\alpha K_{+}-\alpha^{*} K_{-}\right)=\exp \left(\sigma_{1} K_{+}\right) \cdot \exp \left(\sigma_{2} K_{0}\right) \cdot \exp \left(\sigma_{3} K_{-}\right) .
$$

Rewriting $\alpha=\lambda \cdot \mathrm{e}^{i \theta}$ with $\lambda \geq 0$ and $0 \leq \theta<2 \pi$, the exact solutions ${ }^{7}$ are given by

$$
\sigma_{1}=\mathrm{e}^{i \theta} \tanh (\lambda), \quad \sigma_{2}=-2 \log (\cosh (\lambda)), \quad \sigma_{3}=-\mathrm{e}^{-i \theta} \tanh (\lambda) .
$$

Table III presents numerical results of the disentanglement coefficients using the $\mathrm{BCH}$ approximation.

\section{SU(2) algebra}

The SU(2) Lie algebra has three generators $\left\{J_{+}, J_{0}, J_{-}\right\}$, which satisfy the commutation relations

$$
\left[J_{+}, J_{0}\right]=-J_{-}, \quad\left[J_{+}, J_{-}\right]=2 J_{0}, \quad\left[J_{0}, J_{-}\right]=-J_{-} .
$$

Here, we want to calculate the disentanglement

$$
\exp \left(\alpha J_{+}-\alpha^{*} J_{-}\right)=\exp \left(\sigma_{1} J_{+}\right) \cdot \exp \left(\sigma_{2} J_{0}\right) \cdot \exp \left(\sigma_{3} J_{-}\right) .
$$

Rewriting again $\alpha=\lambda \cdot e^{i \theta}$, the exact solutions as derived in Refs. 7 and 9 are 
TABLE III. Comparison of exact and approximate disentangling coefficients for the SU(1,1) algebra defined in Sec. IV C.

\begin{tabular}{cccc}
\hline \hline$p$ & $\alpha$ & $\left\{\left\|\tau_{1}-\sigma_{1}\right\|, \ldots,\left\|\tau_{3}-\sigma_{3}\right\|\right\}$ & CPU time (s) \\
\hline 4 & 1 & $\left\{1.28 \times 10^{-2}, 3.72 \times 10^{-2}, 6.70 \times 10^{-3}\right\}$ & 0.1 \\
8 & 1 & $\left\{4.89 \times 10^{-4}, 1.40 \times 10^{-3}, 2.81 \times 10^{-4}\right\}$ & 0.7 \\
12 & 1 & $\left\{2.19 \times 10^{-5}, 6.31 \times 10^{-5}, 1.29 \times 10^{-5}\right\}$ & 5.6 \\
4 & $1+\frac{i}{2}$ & $\left\{1.91 \times 10^{-2}, 6.63 \times 10^{-2}, 7.39 \times 10^{-3}\right\}$ & 0.1 \\
8 & $1+\frac{i}{2}$ & $\left\{1.07 \times 10^{-3}, 3.64 \times 10^{-3}, 4.96 \times 10^{-4}\right\}$ & 0.8 \\
12 & $1+\frac{i}{2}$ & $\left\{7.23 \times 10^{-5}, 2.48 \times 10^{-4}, 3.48 \times 10^{-5}\right\}$ & 6.4 \\
4 & $\frac{1}{2}+i$ & $\left\{1.91 \times 10^{-2}, 6.63 \times 10^{-2}, 7.39 \times 10^{-3}\right\}$ & 0.1 \\
8 & $\frac{1}{2}+i$ & $\left\{1.07 \times 10^{-3}, 3.64 \times 10^{-3}, 4.96 \times 10^{-4}\right\}$ & 0.9 \\
12 & $\frac{1}{2}+i$ & $\left\{7.23 \times 10^{-5}, 2.48 \times 10^{-4}, 3.48 \times 10^{-5}\right\}$ & 6.4 \\
\hline \hline
\end{tabular}

$$
\sigma_{1}=\mathrm{e}^{i \theta} \tan (\lambda), \quad \sigma_{2}=2 \log (\sec (\lambda)), \quad \sigma_{3}=-\mathrm{e}^{-i \theta} \tan (\lambda) .
$$

Numerical results can be found in Table IV.

Moreover, Fig. 2 presents some results for $\alpha \in \mathbb{R}$ and $0 \leq \alpha \leq 1.5$. The exact solutions $\sigma_{1}$ $=\sigma_{1}(\alpha)$ to $\sigma_{3}=\sigma_{3}(\alpha)$ correspond to the thin black curves and the $\mathrm{BCH}$ approximation for orders $p=3, p=7$, and $p=11$ are illustrated by dotted, dashed, and solid curves, respectively.

\section{E. The six-dimensional double photon algebra}

In our last example we turn to the double photon algebra $\left\{K_{+}, A^{+}, K_{0}, I, K_{-}, A\right\}$, defined by the following commutation relations:

$$
\begin{gathered}
{\left[K_{+}, A^{+}\right]=0, \quad\left[K_{+}, K_{0}\right]=-K_{+}, \quad\left[K_{+}, I\right]=0,} \\
{\left[K_{+}, K_{-}\right]=-2 K_{0}, \quad\left[K_{+}, A\right]=-A^{+}, \quad\left[A^{+}, K_{0}\right]=-\frac{1}{2} A^{+},} \\
{\left[A^{+}, I\right]=0, \quad\left[A^{+}, K_{-}\right]=-A, \quad\left[A^{+}, A\right]=-I,} \\
{\left[K_{0}, I\right]=0, \quad\left[K_{0}, K_{-}\right]=-K_{-}, \quad\left[K_{0}, A\right]=-\frac{1}{2} A,} \\
{\left[I, K_{-}\right]=0, \quad[I, A]=0, \quad\left[K_{-}, A\right]=0 .}
\end{gathered}
$$

With $\alpha=\lambda \cdot \mathrm{e}^{i \theta}$ and $\zeta=\mu \cdot \mathrm{e}^{i \phi}$, our goal is to approximate the disentanglement form

TABLE IV. Comparison of exact and approximate disentangling coefficients for the SU(2) algebra defined in Sec. IV D.

\begin{tabular}{cccc}
\hline \hline$p$ & $\alpha$ & $\left\{\left\|\tau_{1}-\sigma_{1}\right\|, \ldots,\left\|\tau_{3}-\sigma_{3}\right\|\right\}$ & CPU time $(\mathrm{s})$ \\
\hline 4 & 1 & $\left\{5.14 \times 10^{-2}, 9.13 \times 10^{-2}, 9.55 \times 10^{-2}\right\}$ & 0.1 \\
8 & 1 & $\left\{1.19 \times 10^{-2}, 1.23 \times 10^{-2}, 1.51 \times 10^{-2}\right\}$ & 0.4 \\
12 & 1 & $\left\{7.38 \times 10^{-4}, 7.54 \times 10^{-4}, 9.22 \times 10^{-4}\right\}$ & 4.9 \\
4 & $1+\frac{i}{2}$ & $\left\{8.52 \times 10^{-1}, 8.25 \times 10^{-1}, 1.17 \times 10^{-0}\right\}$ & 0.1 \\
8 & $1+\frac{i}{2}$ & $\left\{7.99 \times 10^{-2}, 7.12 \times 10^{-2}, 1.03 \times 10^{-1}\right\}$ & 0.5 \\
12 & $1+\frac{i}{2}$ & $\left\{7.14 \times 10^{-3}, 6.36 \times 10^{-3}, 9.04 \times 10^{-3}\right\}$ & 5.4 \\
4 & $\frac{1}{2}+i$ & $\left\{8.53 \times 10^{-1}, 8.25 \times 10^{-1}, 1.17 \times 10^{-0}\right\}$ & 0.1 \\
8 & $\frac{1}{2}+i$ & $\left\{7.99 \times 10^{-2}, 7.12 \times 10^{-2}, 1.03 \times 10^{-1}\right\}$ & 0.5 \\
12 & $\frac{1}{2}+i$ & $\left\{7.14 \times 10^{-3}, 6.36 \times 10^{-3}, 9.04 \times 10^{-3}\right\}$ & 5.2 \\
\hline \hline
\end{tabular}




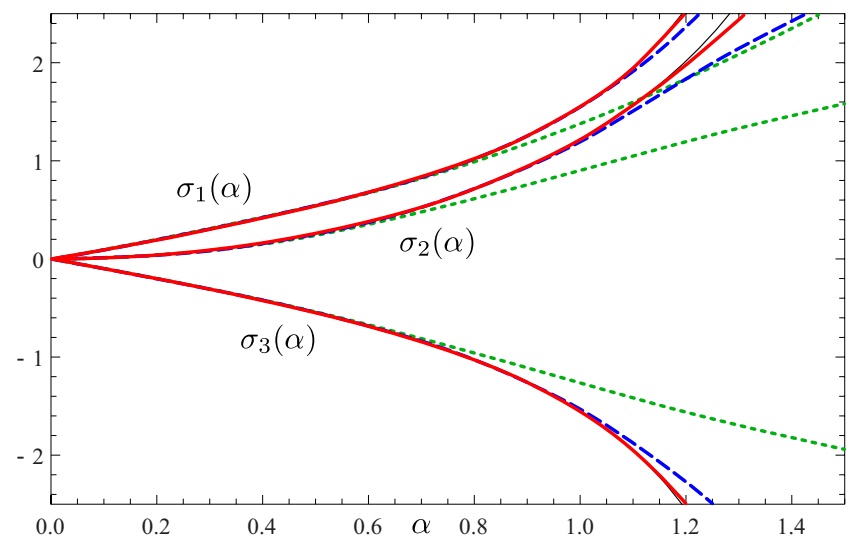

FIG. 2. (Color online) Comparison of exact and approximate disentangling coefficients for the SU(2) algebra defined in Sec. IV D for $0 \leq \alpha \leq 1.5$. Exact solutions (thin black curves), approximation order $p=3$ (dotted green curves), approximation order $p=7$ (dashed blue curves), and approximation order $p=11$ (solid red curves).

$$
\begin{gathered}
\exp \left(\alpha K_{+}+\zeta A^{+}-\alpha^{*} K_{-}-\zeta^{*} A\right) \\
=\exp \left(\sigma_{1} K_{+}\right) \cdot \exp \left(\sigma_{2} A^{+}\right) \cdot \exp \left(\sigma_{3} K_{0}\right) \cdot \exp \left(\sigma_{4} I\right) \cdot \exp \left(\sigma_{5} K_{-}\right) \cdot \exp \left(\sigma_{6} A\right) .
\end{gathered}
$$

The exact coefficients as given in Ref. 9 are

$$
\begin{gathered}
\sigma_{1}=\mathrm{e}^{i \theta} \tanh (\lambda), \\
\sigma_{2}=-\frac{\mu}{\lambda \cosh (\lambda)} \cdot\left((\cosh (\lambda)-1) \cdot \mathrm{e}^{i(\theta-\phi)}-\sinh (\lambda) \cdot \mathrm{e}^{i \phi}\right), \\
\sigma_{3}=-2 \log (\cosh (\lambda)), \\
\sigma_{4}=-\frac{\mu^{2}}{\lambda^{2} \cosh (\lambda)} \cdot((\cosh (\lambda)-1)+i \sin (\theta-2 \phi) \cdot(\sinh (\lambda)-\lambda \cosh (\lambda))), \\
\sigma_{5}=-\mathrm{e}^{-i \theta} \tanh (\lambda), \\
\sigma_{6}=-\frac{\mu}{\lambda \cosh (\lambda)} \cdot\left((\cosh (\lambda)-1) \cdot \mathrm{e}^{-i(\theta-\phi)}+\sinh (\lambda) \cdot \mathrm{e}^{-i \phi}\right) .
\end{gathered}
$$

We believe that the result for $\sigma_{6}$ given in Ref. 9 has a misprint for the sign before the sinh term. Finally, results using the $\mathrm{BCH}$ approximation are presented in Table V.

\section{CONCLUSION}

In this paper, we suggested a general method for the approximate disentangling of exponential operators that satisfy a finite-dimensional Lie algebra. We provide a computer implementation that determines the disentangling coefficients $\sigma$ as defined in Eq. (1) for, in principle, arbitrary Lie algebras. The method uses the $\mathrm{BCH}$ theorem in an essential way, and therefore, we expect that it converges only in a finite convergence radius centered at zero for all parameters of a given problem. The limits of convergence have not been investigated in detail.

The computer implementation provided is a basic demonstration of the method, but does not control accuracy by, e.g., comparing different approximation orders. Accuracy control could easily be added in a more sophisticated version of the implementation. Furthermore, the code is optimized for simplicity, but not for running time.

Five numerical examples with known exact analytic solutions demonstrate that the method yields very good approximations for various parameter sets. For example, we obtained a quite good approximate disentangling for the four-dimensional Lie algebra as defined in Sec. IV B already for an approximation order $p=8$. For the three-dimensional $\mathrm{SU}(2)$ algebra, the same accuracy was achieved for an order of $p \geq 14$. 
TABLE V. Comparison of exact and approximate disentangling coefficients for the double photon algebra defined in Sec. IV E.

\begin{tabular}{cccr}
\hline \hline$p$ & $\{\alpha, \zeta\}$ & $\left\{\left\|\tau_{1}-\sigma_{1}\right\|, \ldots,\left\|\tau_{6}-\sigma_{6}\right\|\right\}$ & CPU time (s) \\
\hline 4 & $\{1,1\}$ & $\left\{1.28 \times 10^{-2}, 6.86 \times 10^{-3}, 3.72 \times 10^{-2}, 1.86 \times 10^{-3}, 6.71 \times 10^{-3}, 7.00 \times 10^{-3}\right\}$ & 0.2 \\
8 & $\{1,1\}$ & $\left\{4.89 \times 10^{-4}, 2.44 \times 10^{-4}, 1.40 \times 10^{-3}, 6.04 \times 10^{-5}, 2.81 \times 10^{-4}, 2.84 \times 10^{-4}\right\}$ & 43.8 \\
12 & $\{1,1\}$ & $\left\{2.19 \times 10^{-5}, 1.11 \times 10^{-5}, 6.31 \times 10^{-5}, 2.92 \times 10^{-6}, 1.29 \times 10^{-5}, 1.25 \times 10^{-5}\right\}$ & 6349.4 \\
4 & $\left\{1+\frac{i}{2}, \frac{1}{2}+i\right\}$ & $\left\{1.91 \times 10^{-2}, 2.03 \times 10^{-2}, 6.63 \times 10^{-2}, 1.43 \times 10^{-2}, 7.39 \times 10^{-3}, 3.04 \times 10^{-2}\right\}$ & 0.3 \\
8 & $\left\{1+\frac{i}{2}, \frac{1}{2}+i\right\}$ & $\left\{1.07 \times 10^{-3}, 1.16 \times 10^{-3}, 3.64 \times 10^{-3}, 6.81 \times 10^{-4}, 4.96 \times 10^{-4}, 1.54 \times 10^{-3}\right\}$ & 51.8 \\
12 & $\left\{1+\frac{i}{2}, \frac{1}{2}+i\right\}$ & $\left\{7.23 \times 10^{-5}, 7.93 \times 10^{-5}, 2.46 \times 10^{-4}, 4.62 \times 10^{-5}, 3.48 \times 10^{-5}, 1.02 \times 10^{-4}\right\}$ & 6581.2 \\
4 & $\left\{\frac{1}{2}+\frac{3 i}{7},-\frac{1}{2}+\frac{2 i}{3}\right\}$ & $\left\{2.54 \times 10^{-3}, 3.94 \times 10^{-3}, 4.00 \times 10^{-3}, 3.15 \times 10^{-3}, 2.05 \times 10^{-3}, 3.22 \times 10^{-3}\right\}$ & 0.2 \\
8 & $\left\{\frac{1}{2}+\frac{3 i}{7},-\frac{1}{2}+\frac{2 i}{3}\right\}$ & $\left\{2.07 \times 10^{-5}, 3.28 \times 10^{-5}, 3.28 \times 10^{-5}, 2.64 \times 10^{-5}, 1.71 \times 10^{-5}, 2.40 \times 10^{-5}\right\}$ & 44.8 \\
12 & $\left\{\frac{1}{2}+\frac{3 i}{7},-\frac{1}{2}+\frac{2 i}{3}\right\}$ & $\left\{1.91 \times 10^{-7}, 3.03 \times 10^{-7}, 3.02 \times 10^{-7}, 2.44 \times 10^{-7}, 1.58 \times 10^{-7}, 2.16 \times 10^{-7}\right\}$ & 6366.3 \\
\hline \hline
\end{tabular}

As can be seen from our numerical results presented in Sec. IV, the CPU times and the accessible orders of approximation strongly depend on the dimension of the given Lie algebra. For four-dimensional algebras, the approximation order 12 can be obtained in a few seconds. On the other hand, in higher-dimensional algebras the required CPU times increase rapidly as can be seen, e.g., in Table V. However, for specific applications, much more efficient implementations of the proposed method could be possible.

As explained in the text, we need to pick out from the set of solutions of a system of nonlinear equations the one that fulfills a certain boundary condition. This is done in several steps starting from a trivial initial auxiliary problem. The number of auxiliary problems to be considered is controlled by the parameter $M$ to be given to our program. Although we used $M=10$ in all numerical examples, there might be problems for which our method picks the correct solution only for some larger values of $M$. This would need to be carefully controlled by suitable tests.

\footnotetext{
${ }^{1}$ J. Wei and E. Norman, J. Math. Phys. 4, 575 (1963).

${ }^{2}$ R. P. Feynman, Phys. Rev. 84, 108 (1951).

${ }^{3}$ R. J. Glauber, Phys. Rev. 84, 395 (1951).

${ }^{4}$ R. M. Wilcox, J. Math. Phys. 8, 962 (1967).

${ }^{5}$ V. S. Popov, Phys. Usp. 50, 1217 (2007).

${ }^{6}$ M. Suzuki, Commun. Math. Phys. 57, 193 (1977).

${ }^{7}$ A. Mufti, H. A. Schmitt, and M. Sargent III, Am. J. Phys. 61, 729 (1993).

${ }^{8}$ R. Barak and Y. Ben-Aryeh, J. Opt. Soc. Am. B 25, 361 (2008).

${ }^{9}$ A. DasGupta, Am. J. Phys. 64, 1422 (1996).

${ }^{10}$ H. F. Baker, Proc. London Math. Soc. s2-3, 24 (1905).

${ }^{11}$ J. E. Campbell, Proc. London Math. Soc. s1-29, 14 (1897)

${ }^{12}$ F. Hausdorff, Ber. Verh. Saechs. Akad. Wiss. Leipzig, Math.-Phys. K1. 58, 19 (1906).

${ }^{13}$ M. Weyrauch and D. Scholz, Comput. Phys. Commun. 180, 1558 (2009).

${ }^{14}$ E. B. Dynkin, Mat. Sb. 67, 155 (1949).

${ }^{15}$ E. B. Dynkin, Dokl. Akad. Nauk SSSR 57, 323 (1947); an English translation may be found in Selected Papers of E.B. Dynkin with Commentary, edited by E. B. Dynkin, A. A. Yushkevich, G. M. Seitz, and A. L. Onishchik (American Mathematical Society, Providence, RI/International Press, Cambridge, MA, 2000).

${ }^{16}$ J. A. Oteo, J. Math. Phys. 32, 419 (1991).

${ }^{17}$ Wolfram Research, Inc., MATHEMATICA, Version 7.0, Champaign, Illinois (2008)

${ }^{18}$ K. Goldberg, Duke Math. J. 23, 13 (1956).

${ }^{19}$ M. Toutounji, J. Chem. Phys. 128, 164103 (2008).
} 